\title{
LA GESTIÓN DEL CONOCIMIENTO EN LOS PROCESOS DE CALIDAD DE LA EDUCACIÓN SUPERIOR
}

Autor:

Alexis Alfredo Tamayo Chacón ${ }^{1}$

Dirección para correspondencia: alextama50@gmail.com

Fecha de recepción: 18 de abril de 2020

Fecha de aceptación: 25 de julio de 2020

Fecha de publicación: 4 de septiembre de 2020

Citación/como citar este artículo: Tamayo, A. (2020). La gestión del conocimiento en los procesos de calidad de la educación superior. Rehuso, 5(3), 1-15. Recuperado de: https://revistas.utm.edu.ec/index.php/Rehuso/article/view/1684

\section{Resumen}

La gestión de conocimiento en las universidades coadyuva al acceso de la sociedad de la información, único camino para llevar a un país a la sociedad del conocimiento y reducir las brechas de desigualdad. Con esta investigación se busca promover la reflexión teórica entre modelos de gestión del conocimiento y la calidad educativa universitaria en el Ecuador. Se realiza una exhaustiva revisión documental de los fundamentos teóricos de la gestión del conocimiento y de la calidad en la educación superior en el contexto nacional justo en el último período de evaluación de estas instituciones (20182020). Sustenta una acepción epistemológica positivista que permitió descubrir cómo funciona la gestión del conocimiento en los procesos de calidad educativa universitaria y como se afectan significativamente de forma proporcional. Ejecuta un acercamiento explicativo (no experimental) basado en la obtención de datos cualitativos mediante la observación, con un alcance nacional. Se identificó que, la calidad en las instituciones de educación superior, será proporcional a la capacidad de respuesta de estas a los actuales y futuros retos que le impone la sociedad. Los sistemas de gestión de conocimiento son indispensable para que estás instituciones mejoren su adaptabilidad al entorno y su capacidad de respuesta a los contextos imperantes. La obtención de estas conclusiones se basa en el método inductivo a partir de la observación de eventos reales que nos permitieron establecer predicciones o generalidades, observando de manera transversal las situaciones en un mismo período.

Palabras clave: Gestión del Conocimiento; Enseñanza Superior; Calidad de la Educación; Procesamiento de la Información; Información y Comunicación

\section{KNOWLEDGE MANAGEMENT IN QUALITY PROCESSES OF HIGHER EDUCATION}

\begin{abstract}
Knowledge management in universities contributes to access to the information society, the only way to lead a country to the knowledge society and reduce inequality gaps. This research seeks to promote theoretical reflection between models of knowledge management and university educational quality in Ecuador. An exhaustive documentary review of the theoretical foundations of knowledge management and quality in higher education in the national context is carried out in the last evaluation period of these institutions (2018-2020). It supports a positivist epistemological meaning that allowed discovering how knowledge management works in the processes of university educational quality and how they are significantly affected in a proportional way. It executes an explanatory (non-
\end{abstract}

\footnotetext{
${ }^{1}$ Instituto Superior Tecnológico Bernardo O’Higgins; Quito, Ecuador. Correo electrónico: alextama50@gmail.com
} 
experimental) approach based on obtaining qualitative data through observation, with a national scope. It was identified that the quality of higher education institutions will be proportional to their ability to respond to current and future challenges imposed by society. Knowledge management systems are essential for these institutions to improve their adaptability to the environment and their ability to respond to prevailing contexts. Obtaining these conclusions is based on the inductive method from the observation of real events that allowed us to establish predictions or generalities, observing transversally the situations in the same period.

Keywords: Knowledge Management; Higher Education; Quality of Education; Information Processing; Information and Communication

\section{Introducción}

La calidad universitaria en América Latina empezó a tratarse con fuerza en los 90’; fue entonces que se crearon organismos de control en México (1989), Chile (1990), Colombia (1992), Brasil (1993), Argentina (1995/96) y así sucesivamente hasta llegar a los años 2000.

En diferentes certámenes a nivel mundial se hace hincapié en la necesidad de tener una educación superior de calidad dado los desafíos presentes y futuros que enfrenta la sociedad actual; las universidades nos pueden revelar y ofrecer el conocimiento necesario para entender estos fenómenos. La gestión del conocimiento se proclama como un mecanismo necesario para administrar de manera inteligente recursos humanos y técnicos, aplicable a cualquier organización por lo que resulta incuestionable que la gestión educativa en las universidades esté sujeta a la gestión del conocimiento "por ser estas agentes contemporáneos en la producción y transmisión de conocimientos" (Naranjo, 2016, p. 141). La gestión del conocimiento fomenta entornos inteligentes y sensibles que implican revisar y criticar prácticas de la vida universitaria para lo que necesita, de manera indispensable, una "cultura del conocimiento" que permita subordinar las culturas burocráticas y tribales a un macro proyecto al servicio común o social.

Para alcanzar estándares de calidad, la universidad, debe resolver "problemas como tecnología, globalización, acceso y participación; considerar nuevas formas de aprendizaje y formación de nuevos académicos; acometer reformas organizacionales y de financiamiento" (García, 2016), punto de partida para que la educación superior (universidades y politécnicos) integre una red donde se pueda gestionar todo el conocimiento creado en ellas.

Es por ello que la Ley Orgánica de Educación Superior (LOES) (2018), en su artículo 93 define los principios de calidad como:

... la búsqueda continua, auto-reflexiva del mejoramiento, aseguramiento y construcción colectiva de la cultura de la calidad educativa superior con la participación de todos los estamentos de las instituciones de educación superior y el Sistema de Educación Superior, basada en el equilibrio de la docencia, la investigación e innovación y la vinculación con la sociedad, orientadas por la pertinencia, la inclusión, la democratización del acceso y la equidad, la diversidad, la autonomía responsable, la integralidad, la democracia, la producción de conocimiento, el diálogo de saberes, y valores ciudadanos (p.40).

Pero, como podríamos enfocarnos en la "producción del conocimiento" si no tenemos un adecuado sistema de gestión de conocimiento en nuestras instituciones de educación superior. La calidad, como concepto, depende en gran medida de los indicadores que la sociedad nos impone; no podemos hablar de una sociedad del conocimiento si nuestras universidades a través de su misión (producción, 
trasmisión y transferencia de conocimientos) no son capaces de integrase a esta, ya sea a través de la investigación, actividades docentes o comunitarias.

\section{Metodología}

El presente se centra en develar limitaciones de los modelos de evaluación a las instituciones de educación superior en nuestro país basado en estrategias o mecanismos relacionados a la gestión del conocimiento por lo que se considera una investigación aplicada y, según el nivel de profundización de este tema, la consideramos explicativa ya que encontramos causas y demostramos consecuencias de la correlación de ambas variables (gestión del conocimiento; calidad de la educación superior).

Según el tipo de datos utilizados es cualitativa y no experimental basada fundamentalmente en el análisis documental y la observación dado que los datos son un tanto subjetivos según su descripción pero objetivos teniendo en cuenta los resultados del análisis; las fuentes de información utilizadas fueron básicamente modelos de evaluación implementados por los organismos de control de calidad de la educación superior en el Ecuador, en el período 2018-2020, aunque también se analizaron modelos anteriores (2014 y 2017), de la misma forma se revisaron experiencias de implementación de la gestión del conocimiento en Instituciones de Educación Superior de América Latina en la última década, lo que proporcionó la aplicación del método inductivo a partir de la obtención de conclusiones basada en la observación de hechos transversalizando un momento concreto y compartiendo ambas variables en la misma temporalidad.

Se pretende mostrar el análisis de la correlación de nuestras variables a partir de cuatro puntos de vistas:

1. Las Instituciones de Educación Superior (IES)

2. Los organismos de control

3. Las Tecnologías de la Información y las Comunicaciones (TIC's)

4. El profesional de la información

El análisis tiene varios factores de cuestionamiento teniendo en cuenta que estamos relacionando el uso (o no) de los sistemas de conocimiento y su influencia en la calidad de la educación superior en las IES de nuestro país.

\section{Resultados}

Estos cuatro puntos de vistas los relacionamos a partir de su vínculo e interrelación. Las IES son regidas por los organismos de control pero consideramos insuficiente las valoraciones del uso de las TIC’s y la valía del profesional de la información en sus modelos evaluativos.

\subsection{Las Instituciones de Educación Superior (IES)}

Como resultado de la docencia universitaria y como ejercicios de graduación de carreras o materias, nuestras IES desarrollan programas de investigación, que ciertamente se estructuran y se hacen coincidir con objetivos estratégicos, líneas de investigación e indicadores de calidad; aunque no podemos decir que los puntos fuertes de estas investigaciones sean la vinculación de las IES con el medio externo, por otra parte no siempre es buena la calidad de la actividad académica universitaria debido a débiles sistemas de gestión del conocimiento y una baja inversión en ellos.

En investigaciones revisadas se comprobó el exiguo apoyo de las IES a la producción de conocimientos incluso con la mediación de las bibliotecas, ello lo refleja una muestra de 25 universidades ecuatorianas (entre privadas y públicas) y como pocas apoyan la investigación por 
medio de la institución rectora de los sistemas de gestión del conocimiento (la biblioteca). Los servicios que estas ofrecen están básicamente relacionados con accesos a recursos digitales, sin embargo no se encuentran productos que aporten valor a la información tácita o explicita. Esta distribución se comporta de la siguiente manera:

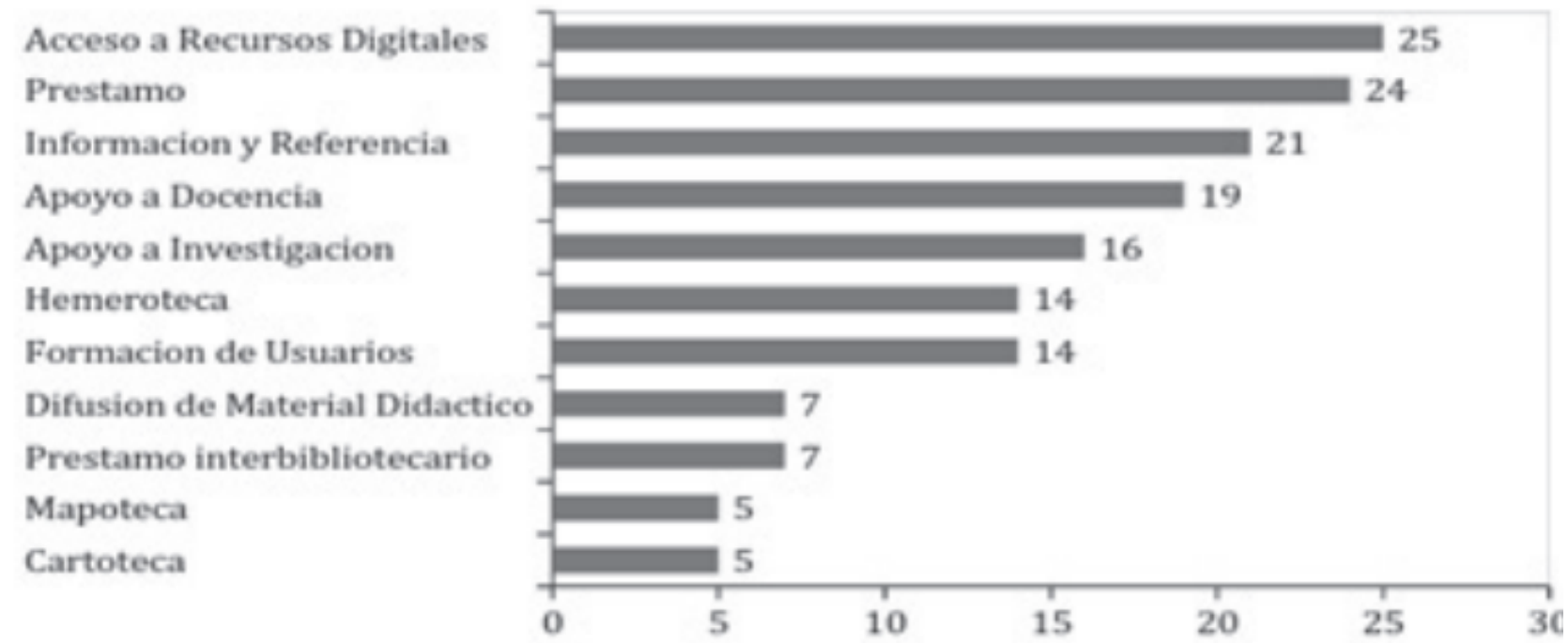

Figura 1 Servicios que disponen las bibliotecas universitarias. Recuperado de Análisis longitudinal del sistema bibliotecario ecuatoriano.

La interconexión de bibliotecas universitarias tradicionalmente se ha basado en el intercambio de bibliografía y la compra de recursos electrónicos como software, bases de datos y propuestas de valor para los miembros, sin embargo, el compromiso debe darse en transformar en servicios los resultados de estudiantes y profesores, además de los objetivos y prioridades institucionales.

\section{Los organismos de control}

Sabemos que gestionar conocimiento es un coeficiente productivo, sobre todo en las IES, pero lo que no sabemos es si los sistemas que se poseen son idóneos. Lo que quiere decir que el conocimiento "especializados" debe estar integrado a propósitos establecidos. Se podría preguntar entonces si este coeficiente productivo está asociado a un sistema de gestión de conocimiento en nuestras universidades.

Las regulaciones derivadas de los organismos de control y que se relacionan de forma directa con las bibliotecas, como centro rector de la gestión del conocimiento institucional, no cumplen con las expectativas de evaluar un sistema integral de calidad educativa si tenemos en cuenta que ninguna de ellas responde a la gestión del conocimiento:

Tabla 1: Regulaciones de los organismos de control para con las bibliotecas

\begin{tabular}{|c|c|c|c|c|}
\hline Año & Org. de control & Documento & Articulo & Refiere \\
\hline & Consejo de & & & \\
\hline $\begin{array}{l}\text { Última } \\
\text { modificación: } \\
\text { 02-ago.-2018 }\end{array}$ & $\begin{array}{l}\text { Aseguramiento } \\
\text { de la Calidad de } \\
\text { la Educación } \\
\text { Superior } \\
\text { (CACES) }\end{array}$ & $\begin{array}{l}\text { Ley Orgánica } \\
\text { de Educación } \\
\text { Superior }\end{array}$ & $\begin{array}{l}\text { Capítulo 2, } \\
\text { artículo } 109, \\
\text { apartado } 11\end{array}$ & $\begin{array}{l}\text { Contar con bibliotecas, hemerotecas, } \\
\text { videotecas y más recursos técnicos pedagógicos } \\
\text { que garanticen un eficiente aprendizaje }\end{array}$ \\
\hline $\begin{array}{l}\text { Última } \\
\text { modificación: } \\
\text { 02-ago.-2018 }\end{array}$ & CACES & $\begin{array}{l}\text { Ley Orgánica } \\
\text { de Educación } \\
\text { Superior }\end{array}$ & Artículo 143 & $\begin{array}{l}\text { Las instituciones de educación superior } \\
\text { públicas y particulares desarrollarán e } \\
\text { integrarán sistemas interconectados de } \\
\text { bibliotecas a fin de promover el acceso }\end{array}$ \\
\hline
\end{tabular}


RPC-SO-08-

No. $111-2019$

$\begin{array}{ccl} & & \text { Modelo de } \\ & & \text { Evaluación } \\ \text { Externa de } \\ \text { Universidades } \\ \text { Junio 2019 Escuelas } \\ & & \text { CACES } \quad \\ & & \text { Politécnicas } \\ & 2019\end{array}$

igualitario a los acervos existentes, y facilitar préstamos e intercambios bibliográficos. Participarán en bibliotecas digitales y sistemas de archivo en línea de publicaciones académicas a nivel mundial.

La institución dispone de bibliotecas que cuentan con instalaciones apropiadas, con un

Estándar 17: acervo bibliográfico físico y digital acorde a la Bibliotecas oferta académica, con un sistema informático de gestión que responde a los requerimientos de la comunidad universitaria.

a) Centro de apoyo.- El centro de apoyo deberá contar con una adecuada infraestructura física, tecnológica y pedagógica, que facilite el acceso de los estudiantes a bibliotecas físicas $\mathrm{y}$ virtuales. De igual manera, deberá asegurar condiciones para que la planta académica gestione los distintos componentes del Artículo 77, aprendizaje, cuando fuere el caso. Este incisos a) $y$ distancia.

b)

b) Bibliotecas virtuales.- Acceso abierto al menos a una biblioteca virtual y un repositorio digital de apoyo para sus estudiantes. La biblioteca incluirá recursos básicos para las actividades obligatorias de la oferta académica y recursos complementarios que permitan la profundización, ampliación o especialización de los conocimientos.

Modelo de

Evaluación Institucional para los Institutos

2020 CACES Superiores Técnicos Tecnológicos en Proceso de Acreditación 2020
Criterio 5: El instituto dispone de una biblioteca acord Recursos e e a la oferta académica, así como con infraestructu instalaciones, recursos, personal y un sistema i ra (Subcriterio 5.1: Biblioteca) nformático específico que permita su gestión. Además, debe responder a los requerimientos de las carreras, profesores y estudiantes para realizar las actividades académicas.

Fuente: elaboración propia

Cierto es que normamos la incorporación obligatoria de las bibliotecas en nuestras IES en el capítulo 2, articulo 109, apartado 11 de la LOES, pero en el artículo 143 se circunscribe su misión a "desarrollar e integrar sistemas de bibliotecas" con el fin de facilitar accesos, prestamos e intercambios bibliográficos. Dejando fuera un sinnúmero de instituciones de información e investigación y circunscribiendo la gestión del conocimiento a la promoción de los acervos existentes y sin tener en cuenta el nuevo conocimiento.

Se acota además que "participarán en bibliotecas digitales y sistemas de archivo en línea de publicaciones académicas a nivel mundial" (CACES, 2018), pero ello no garantiza un sistema de gestión de conocimiento capaz de aportar a la calidad de la educación superior ya que sólo tiene en cuenta el conocimiento explicito dejando fuera el conocimiento tácito.

Sólo por tomar el más actual, el "Modelo de Evaluación Institucional para los Institutos Superiores Técnicos y Tecnológicos en Proceso de Acreditación 2020", propuesto por el CACES (2020), no se tiene en cuentas las diferencias entre bibliotecas, electrónicas, digitales y virtuales; se podría trabajar 
en llevar nuestras colecciones a un catálogo digital sin embargo ello no determina un correcto procesamiento de la información; de la misma forma se exige acceso a bases de datos virtuales sin conocer indicadores de calidad como la especialización temática, grado de cobertura (alcance) o calidad de la información, accesibilidad de documentos, actualidad, crecimiento y fiabilidad de los datos entre otros. La adquisición de bibliotecas virtuales tiene ventajas sustanciales en cambio no garantiza al usuario seguir el ritmo del cambio de la tecnología además de reducir las relaciones de tipo social y no contar en todos los casos con un respaldo físico, no obstante es cierto que la interconexión de estas "bibliotecas" entre las instituciones de educación superior aporta excelencia al servicio pero no podemos hablar de calidad si no añadimos el "valor agregado" personalizado a cada uno de nuestros usuarios.

\section{Las Tecnologías de la Información y las Comunicaciones (TIC's)}

El incremento de las tecnologías no garantiza el uso correcto de las mismas; las TIC's no son el fin sino la herramienta aplicada a la gestión de conocimiento y no sólo para estudiantes sino a todos los procesos de la comunidad universitaria integrando funciones y tareas para la creación de productos y servicios adaptables a las necesidades de conocimiento que garanticen la calidad de la educación superior.

A pesar de que las TIC's se han generalizado hoy en día no podemos decir que haya equidad entre nuestras instituciones de educación superior con respecto al tratamiento de la información; la incorrecta aplicación de un sistema de gestión de conocimientos agudiza las diferencias entre ellas, en cambio un adecuado sistema ayuda a eliminar dichas inequidades y aumentar la calidad de estas instituciones con independencia de la modalidad que ellas sigan. Dadas las características propias de los sistemas de gestión de conocimientos está comprobada su influencia directa en las economías de las universidades de forma positiva las que deben verle como una inversión y saber sacarle el máximo provecho. Las instituciones capaces de aplicar un adecuado sistema de conocimiento se diferenciaran cualitativa y cuantitativamente de su competencia.

En el modelo de evaluación mencionado anteriormente (Modelo de Evaluación Institucional para los Institutos Superiores Técnicos y Tecnológicos en Proceso de Acreditación 2020) entendemos la Interacción virtual como la relación de las TIC's con el entorno universitario aunque hay otras subcriterios que relacionan el tema:

Tabla 2: Regulaciones de los organismos de control para los profesionales de las bibliotecas de las IES

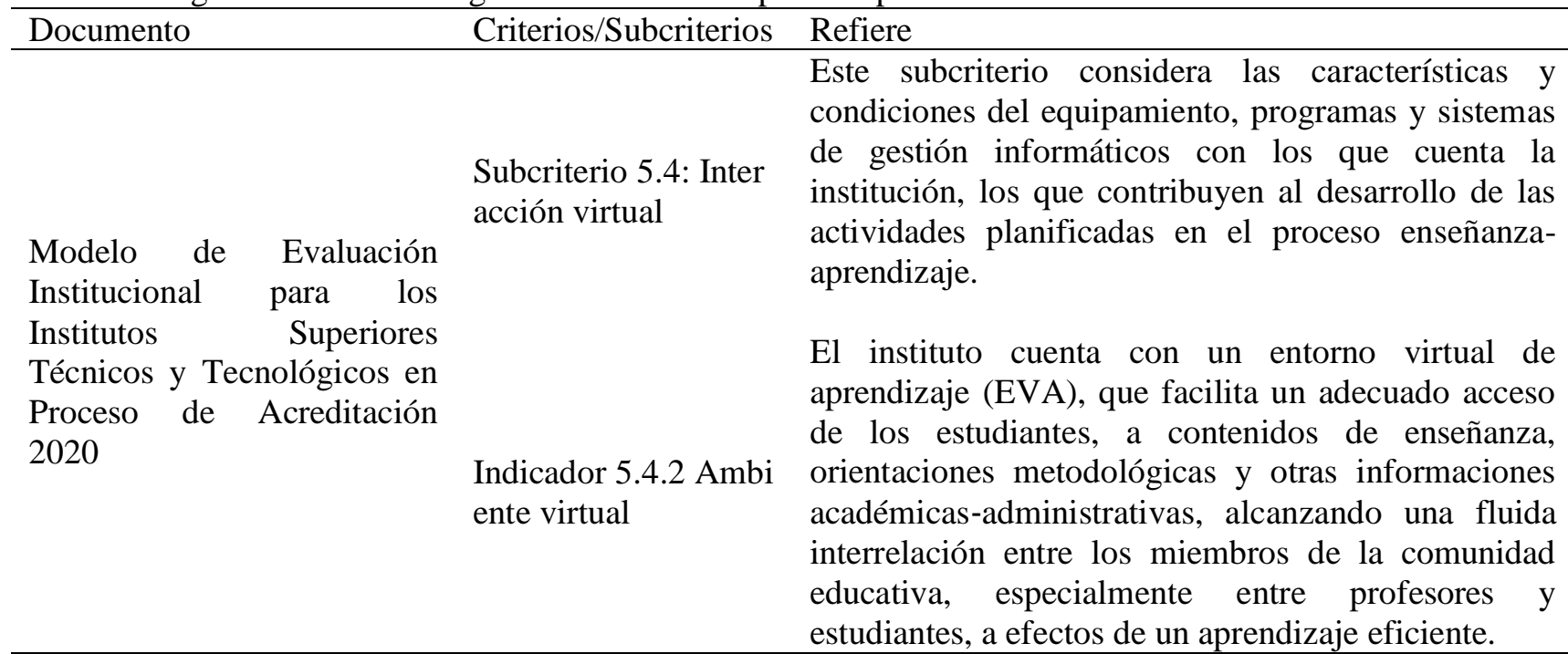


Tanto el subcriterio como el indicador intentan garantizar la forma y no en el contenido, es por ello que se considera dentro de un criterio (Recursos e infraestructura) que, casualmente también encontramos la biblioteca.

El CACES (organismos de control encargado) en sus Respuestas sobre el proceso de evaluación externa para los Institutos Superiores Técnicos y Tecnológicos (2020), se le pregunta acerca de los parámetro que debe tener el sistema informático de una biblioteca en nuestras IES, obteniendo como respuesta que alcanzará con que este pueda, “... facilitar el acceso al acervo bibliográfico y permitir el seguimiento del uso de los recursos bibliográficos físicos y digitales.” (CACES, 2020, p.42); por lo que nos permitimos pensar que con que se pueda acceder al inventario para realizar reservas y prestamos bastaría. Dejando fuera los procesos para la diseminación selectiva de la información y demás servicios no solo al área académica sino también administrativa, convirtiéndose en referente para la gestión de todo el conocimiento institucional.

\section{El profesional de la información}

Un número importante de países ya cuentan con procedimientos para asegurar la calidad en la educación superior. La "sociedad del conocimiento" ha marcado al ser humano como la principal fuerza productiva capaz de generar, procesar y transmitir el conocimiento como fuentes productivas y de poder por lo que se hace necesario asegurar la implementación de estos mecanismos de calidad que incrementen nuestros niveles de confianza.

No es casual que los países con altos índices de desarrollo (Estados Unidos, Reino Unido, Canadá, Australia, Japón, entre otro) cuenten con universidades que formen, intencionalmente, este tipo de profesionales. A nivel mundial existe un sinfín de estas universidades pero según el Rankin de "Top Universities" 2020 las anteriores tienen un alto nivel. En nuestro país no existen muchas variantes para formar profesionales de tercer nivel en estas especialidades, pudiera ser porque no siempre tiene una salida productiva que respalde la inversión o porque quizás no es una política graduar profesionales con estos perfiles que garanticen la calidad de los procesos de gestión del conocimiento. Solo en la Universidad Técnica de Manabí existe un programa vigente con 9 semestres a vencer.

Nuestras IES deben transformarse según las exigencias de una sociedad regida por el conocimiento por lo que se deben formar profesionales que respondan tanto al entorno (criterios sociales) como a las demandas del mercado ocupacional. En la siguiente tabla observamos dos respuestas de los organismos de control al tema de los profesionales que trabajan en las bibliotecas universitarias y que, en determinado momento justifica con modelos anteriores:

Tabla 3: Regulaciones de los organismos de control para con las bibliotecas

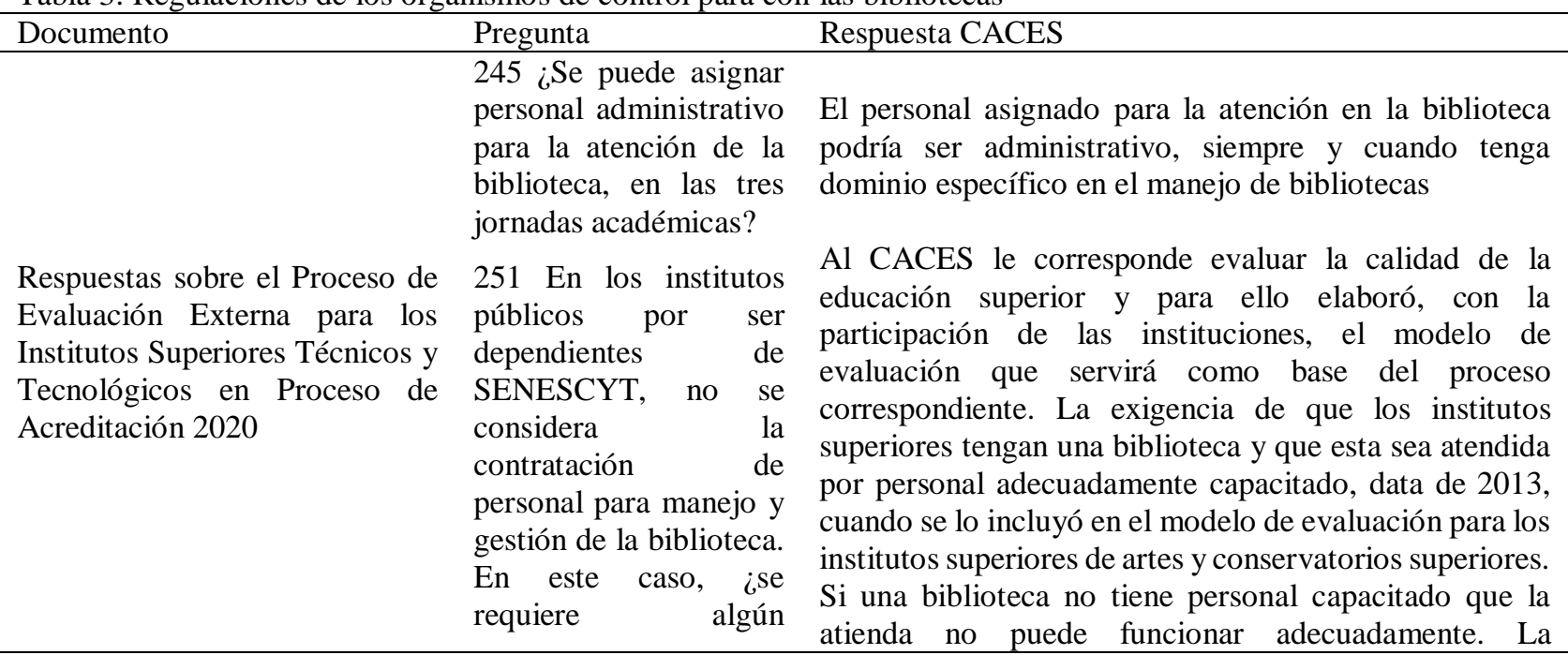


documento justificación? de exigencia de capacitación del personal de la biblioteca no implica un título universitario específico, ni siquiera formación universitaria, sino que, tal como dice el modelo, que el personal cuente con dominio específico en el manejo de la biblioteca. El instituto deberá valorar opciones, dentro de sus condiciones específicas, para cumplir con este requisito.

Fuente: elaboración propia

Los propios Modelos de evaluación de la educación superior minimizan al profesional de la información; el organismos de control encargado (CACES) ha emitido Respuestas sobre el proceso de evaluación externa para los Institutos Superiores Técnicos y Tecnológicos en proceso de acreditación 2020 , donde se preguntaba si se podía asignar “... personal administrativo para la atención de la biblioteca..." (CACES, 2020, p.42), obteniendo la respuesta representada en la tabla y de la que podemos inferir dos cosas; una que no existe personal especializado en el tratamiento de la información, por ende del conocimiento y dos que no importa la formación de un administrativo siempre y cuando sepa manejar una biblioteca. Cabría preguntarnos entonces, ¿se está preparado para asumir los retos de la sociedad del conocimiento en nuestras IES? O podríamos deducir que estos profesionales solo responden al manejo de bibliotecas sin tener en cuenta las dimensiones del tratamiento al conocimiento en nuestras Instituciones (valor añadido de la información explícita, procesos de tratamiento de la información tácita, etc.).

En la segunda respuesta el organismo de control expone que "La exigencia de capacitación del personal de la biblioteca no implica un título universitario específico, ni siquiera formación universitaria, sino que, tal como dice el modelo, que el personal cuente con dominio específico en el manejo de la biblioteca." (CACES, 2020, p.43); por lo que es fácil pensar, sin menos preciar ninguna especialidad, que cualquier persona que sepa manejar una biblioteca, tiene idea para elaborar procesos propios de la gestión de conocimiento en nuestras IES. No en balde los países del primer mundo priorizan y exigen personal altamente especializado para el manejo de los sistemas información de las Instituciones de educación superior, solo así pueden garantizar la calidad de las mismas.

Si valoramos gestionar conocimiento a partir de las bibliotecas como gestoras de información, entonces debemos implicar personal altamente calificado encargado de administrar las diferentes plataformas aportando valor a la información tácita y explicita de toda la institución.

\section{Discusión}

Generalmente, las bibliotecas, en nuestras IES, son dadas a trabajar básicamente en el acceso a la información y están comprometidas con la alfabetización informacional y el aprendizaje, pero no siempre se encuentran a tono con la "Era digital" y sus implicaciones; es difícil avanzar aparejado con los cambios, la financiación es un reto en el cual se debe concientizar para asegurar el valor e impacto de las bibliotecas como gestoras de información y conocimiento en las instituciones de educación superior.

En todo el mundo las universidades se encuentran inmersa en procesos contantes de metaconocimiento ${ }^{1}$ como activos corporativos los cuales residen básicamente en bases de datos, docentes, bibliotecarios, archiveros y personas que, en las instituciones poseen un conocimiento tácito y explícito (García, 2016); lo que pocas han conseguido es interrelacionar el mismo por la falta de un sistema de gestión de conocimientos desde lo individual hasta lo colectivo. Por lo que podemos decir que la gestión del conocimiento no es otra cosa que un proceso creación del conocimiento en sí.

La integración de un sistema de gestión de conocimientos es algo más que la interrelación con una interfaz web de búsqueda, recuperación y consulta de información. El proceso de creación de nuevos 
conocimientos conlleva a las relaciones planificadas, organizadas y estructuradas de los recursos informacionales existentes en el propio sistema; de ellos depende esta nueva forma de creación productiva en nuestras IES y por ende la calidad en todo el sistema de educación superior. Los procesos de integración de los conocimientos se deben realizar en el instante en que la información se introduce al sistema sin obviar el tan importante "valor añadido" de cada una de las demandas de los usuarios. (Docentes, estudiantes o personal administrativo)

En la actualidad se dispones de herramientas que permiten realizar una serie de labores sin necesidad de emplear mucho tiempo. Las plataformas tecnológicas actuales nos permiten hacer consultas de información concreta que complementan con el uso de Bases de Datos y que permiten la elaboración de reportes en cualquier formato.

En las Instituciones se puede ver la gestión del conocimiento como un ente sistémico asumiéndola como "herramienta estratégica que permita investigar, construir, organizar y aplicar conocimientos" (Urdaneta, Villasmil, Palacios, y Prieto, 2019, p.83). Por lo tanto debemos incluirlo en el aprendizaje organizacional de nuestras instituciones de educación superior permitiendo el logro de resultados y la mejora de desempeños. La búsqueda de esta calidad se debe realizar bajo una perspectiva epistemológica en la que se reconozcan las dimensiones del conocer como representación "conocer qué" y del "conocer cómo".

En el 2016 la Red de Archiveros Universitarios de Ecuador (RAUEC) realizó, al CES, una interesante "Propuesta de indicadores para la evaluación institucional del Sistema de Educación Superior" señalando factores fundamentales que no se evaluaban en el antiguo modelo propuesto por el Consejo de Evaluación, Acreditación y Aseguramiento de la Calidad (CEAACES), y que si se definen en las normas de calidad ISO $15489^{2}$, ISO $303004^{3}$ y en la Ley del Sistema Nacional de Archivos (1982); dicha propuesta es un esfuerzo por incluir indicadores que determinan la calidad con que las Instituciones de educación superior gestionan los documentos y la información contenida en ellos.

Según la RAUEC, en casi ninguna de nuestras IES se cuenta con documentos normativos y organizativos que sustente el trabajo documental, tampoco hay uniformidad ni estandarización en el tratamiento de la información (RAUEC, 2016), riesgos que limitan el acceso a la misma y por ende la incapacidad de crear espacios de intercambio de información o creación de conocimiento conjunto, tema medular para alcanzar calidad en la educación superior. La propuesta en sí impulsa la calidad de la educación superior en temas de tratamiento de la información y que serviría para catapultar un adecuado sistema de gestión del conocimiento en nuestras Instituciones. Es imposible hablar de una red de conocimiento o de intercambio si no tenemos normados y estandarizada la información. Propuesta de indicadores que, tanto el CEAACES (en el pasado) como el CACES (en el presente) han hecho caso omiso.

Las instituciones que más conocimiento generan son la universidades es por eso que se hace imprescindible poseer un sistema capaz de estructurar, almacenar y distribuir el conocimiento mediante el uso de las TIC's. Diferentes autores plantean modelos de gestión del conocimiento relacionando las tecnologías, la comunicación, la información y la cultura organizacional cuyo centro gravita en la "Cultura de la Organización"; enfatizan en el factor humano y en lo que denominan "Organizaciones basadas en conocimiento"; estos modelos aplicados a las instituciones de educación superior incluyen elementos capaces de localizar, generar, estructurar, almacenar, transferir, difundir y aplicar el conocimiento con el objetivo de gestionarlos de forma conveniente. En su mayoría coinciden en que estos posean un sistema de información ${ }^{4}$ efectivo, una red colaborativa ${ }^{5}$, espacios de conocimientos ${ }^{6}$ eficientes y un sistema $\mathrm{CRM}^{7}$ interactivo bajo una cultura organizativa que promueva el intercambio de conocimiento y una formación continua según las necesidades de conocimiento de la organización y el entorno. 
Los procesos intrínsecos de una IES (docencia, investigación y extensión universitaria) conllevan una retroalimentación permanente e indispensable que encontramos en los modelos de gestión de conocimiento con entradas (recursos) y salidas (resultados), transformando información tácita en explícita y teniendo en cuenta indicadores de capital intelectual que suministran creación de conocimiento en espiral y que mediante las TIC's viabilizan y apoyan la gestión del conocimiento. La aplicación correcta de estos procesos garantiza calidad en este tipo de instituciones.

La integración de un sistema de gestión de conocimientos es algo más que la interrelación con una interfaz web de búsqueda, recuperación y consulta de información. El proceso de creación de nuevos conocimientos conlleva a las relaciones planificadas, organizadas y estructuradas de los recursos informacionales existentes en el propio sistema; de ellos depende esta nueva forma de creación productiva en nuestras IES y por ende la calidad en todo el sistema de educación superior. Los procesos de integración de los conocimientos se deben realizar en el instante en que la información se introduce al sistema sin obviar el tan importante "valor añadido" de cada una de las demandas de los usuarios. (Docentes, estudiantes o personal administrativo)

En la actualidad se dispones de herramientas que permiten realizar una serie de labores sin necesidad de emplear mucho tiempo. Las plataformas tecnológicas actuales nos permiten hacer consultas de información concreta que complementan con el uso de Bases de Datos y que permiten la elaboración de reportes en cualquier formato.

Los profesionales que componen nuestras IES, específicamente el profesional de la información, deben contribuir al aprendizaje mediante el conocimiento. "La creación y la transformación del conocimiento no son procesos estáticos, debido a que hay que corregir, aprender, enseñar, adecuar e interrelacionarse" (Correa Díaz, Arias Benjumea y Valencia Arias, 2019), por lo que se necesita de personal especializado para guiar los procesos de generación de valor a la conversión de información a conocimiento, ya sea tácito o explícito

Existen varios puntos neurálgicos que atentan en contra la calidad en la educación superior de forma general pero creemos que todo comienza por el profesional de la información que atiende los sistemas de gestión o sencillamente el "bibliotecario", "archivero" o "especialista en sistemas", en fin, el encargado de realizar la selección, evaluación, clasificación, organización y recuperación de la información, además de crear conocimiento y evaluar su impacto.

Es cierto que se han dado pasos con la intención de promover estos sistemas de conocimientos en la educación superior pero la falta de un profesional competente a limitado estos intentos, se intuye que un profesional en sistemas computacionales resuelve el inconveniente y hacemos caso omiso a los procesos de tratamiento de la información y por ende del conocimiento.

Para certificar calidad en la educación superior se debe poseer un adecuado sistema de gestión del conocimiento con profesionales especializados que definan qué información va a gestionar, qué recursos, en qué fuentes, sobre qué actividades o áreas y que valor va a añadir. Cada usuario, sistema o plataforma que se posea tiene necesidades concretas, por ende la asimilación, enlace o compenetración de cada una de ellas es fundamental a la hora de gestionar conocimientos sin importar si es tácito o explicito, ni siquiera si es en papel o electrónico.

Es indispensable tratar el tema de la gestión del conocimiento si no va aparejado a la calidad en la educación superior ya que una depende de la otra, a decir de Naranjo P, González H, y Rodríguez M (2016) esta relación es en sí mismo un indicador de innovación organizacional, reconocido por 
organismos internacionales (Manual de Oslo), considerando la gestión del conocimiento como la inversión inmaterial más relevantes en el mejoramiento de la productividad y el desarrollo de las Instituciones por lo que podríamos preguntarnos si los organismos de control están preparados para asesorar y evaluar dichos sistemas en la búsqueda de calidad en la educación superior.

A la vez coincidimos con Correa Díaz et al.(2019) en que la gestión del conocimiento es una estrategia fundamental para las IES dada la cantidad de información de la que dispone y de la capacidad de transformar "información" en "conocimiento" como elemento clave en la conversión del capital intangible que es.

Se plantea por los autores anteriores que los modelos de gestión de conocimiento, representan las buenas prácticas “... específicamente en las IES, las cuales tienen el papel de ser gestoras de cambio para la creación, difusión, aprendizaje e innovación. Lo anterior justifica la inversión en plataformas, software e infraestructuras que permitan establecer redes para compartir y renovar saberes" (Correa et al., 2019).

Si se entiende tal afirmación que solo los recursos tecnológicos son importantes, estos mismo autores en el propio trabajo hacen énfasis en la dimensión humanista citando que son las personas "más que las tecnologías y los procesos, las agentes de cambio y las generadoras de innovación; es decir, las personas son las creadoras de resultados al analizar los problemas desde diversas perspectivas" (Correa et al., 2019), son estas las que, usando las TIC's, gestionan el conocimiento para la resolución de problemas de las IES de "hoy y del futuro".

En el mismo artículo se señala la subordinación de la gestión del conocimiento a factores como las "capacidades financieras, contexto político y jurídico -en función de la prioridad establecida en las agendas nacionales-, así como las dinámicas sociales y culturales donde se inscribe" (Correa et al., 2019), por lo que es imposible dejar de contextualizar cada IES y considerar además de los aspectos que las transversalizan.

La gestión del conocimiento en las IES se hace mucho más complejo dadas las características a las que responden de tipo social, cultural, moral y pedagógico lo que hace difícil evaluar los niveles de calidad en estas instituciones si tenemos en cuenta que es en estas donde nace la creación científica que le da respuestas lógica a toda una sociedad. La búsqueda de excelencia de los conocimientos requiere un enorme compromiso social y a la vez una responsabilidad ineludible que poseen las universidades y que pueden llevar al éxito o al fracaso un sinnúmero de proyectos individuales y colectivos.

Teniendo en cuenta esta diversidad cada universidad desarrolla modelos de conocimiento diferentes que garantizan su nivel de calidad y pertinencia aunque se sugiere encontrar puntos comunes con otras instituciones de educación superior que permitan el intercambio de conocimientos.

La sociedad de la información implica a la vez un aprendizaje por lo que en ocasiones no determinamos con claridad donde comienza y termina el saber, los tiempos y los sistemas de gestión del conocimiento. En los procesos de enseñanza aprendizaje de nuestras universidades no solo debemos evaluar el uso de las TIC's sino también la transferencia de conocimientos y su impacto, sólo así podríamos aspirar a la calidad de la educación superior.

Por norma general las universidades implementan acciones relacionadas con las TIC's en búsqueda de la calidad, algunas de ellas son la instalación de bibliotecas virtuales, la implementación de sistemas automatizados de tipo académicos y administrativos, el uso de publicaciones electrónicas, programas de educación virtual y la organización de centros de tecnología educativa, todo ello para 
el apoyo de las diferentes áreas de las instituciones (UNESCO, 2009). En la actualidad las universidades basan sus esfuerzos en procesos formativos, de investigación y de extensión cada uno de forma independiente lo que garantiza un funcionamiento tradicional de nuestras instituciones de educación superior. Para garantizar la calidad de estas instituciones es necesaria la optimización de recursos, la reformulación de estructuras y la integración de procesos mediante un acertado sistema de gestión del conocimiento.

En la mayoría de los casos las universidades aplican modelos de gestión de conocimiento con dos enfoques: Capital intelectual ${ }^{8}$ y desde el punto de vista de áreas funcionales que transversalizan los procesos $^{9}$. El modelo más utilizado, y el que sugerimos, en este tipo de institución es el de Ikujiro Nonaka y Hirotaka Takeuchi (como se citó en Huanca, 2013), ya que promueve la creación del conocimiento desde el punto de vista epistemológico y ontológico en el cual interactúan el conocimiento tácito y el explícito, nada más necesario para encontrar la calidad en la educación superior.

\section{En la región (Latinoamérica)}

En nuestra región no existen investigaciones con un nivel de actualidad acorde a lo que esperábamos, luego de realizar una minuciosa búsqueda documental encontramos investigaciones que han trabajado modelos de gestión de conocimiento en pocas universidades como es el caso de la Universidad Nacional Mayor de San Marcos del Perú, de la misma forma hay propuestas como la de Díaz Muñante (2003), dejando planteado como "construir un modelo para la gestión del conocimiento en una realidad educativa universitaria del país, a partir de los procesos de autoanálisis institucional y la construcción de un sistema de indicadores de la calidad educativa..." (Días, 2003, p.5).

En Colombia se encontraron investigaciones en la búsqueda de calidad en las universidades aunque básicamente utilizando las TIC's para incorporar plataformas e-learning. Una de ellas propone un modelo que implica la gestión de conocimiento y capital intelectual el cual elaboraron profesores de la Universidad Tecnológica de Bolívar en Cartagena de Indias donde proponen una herramienta para desarrollar el conocimiento tácito a explícito dentro de una organización (Arraut y Gazabon, 2007). De igual forma Reyes Morales (como se citó en Jama Zambrano V. R., 2019) elabora una investigación sobre la "aplicación de las TIC's en la Gestión del Conocimiento en Instituciones Educativas en Colombia"; este no contempla indicadores de capital intelectual sino que se desarrolla para la gestión académica y el diseño de programas que pretenden dar respuestas a solicitudes de las empresas.

En nuestro país (Ecuador) se ha visto que las empresas no han prestado la suficiente atención a las IES para su progreso, desarrollo incluso investigaciones (I+D) aunque ha sido mutuo pues tampoco las universidades han visto las empresas como caldo de cultivo para sus aprendizajes e innovaciones. León Rodríguez (2015) realiza una investigación mostrando "cómo la gestión del conocimiento organizacional tributa a una mayor adaptabilidad y respuesta de las universidades a los nuevos requerimientos del entorno y los contextos sociales, productivos y culturales" (León Rodríguez, 2015, p.15); en el mismo correlaciona los ejes de la calidad y el sistema de educación superior y cómo con la intervención de sistemas de gestión de procesos, de información, de conocimiento y la interoperabilidad de los mismos se consigue acrecentar los indicadores de calidad en este tipo de instituciones. Otra investigación realizada esta focalizada a la planificación, la realizaron en la Universidad Laica Eloy de Manabí donde se relaciona la gestión del conocimiento con los procesos de planificación, como una de las herramientas necesarias en la toma de decisiones (Jama, Zambrano, Cornejo, y Párraga, 2017, p.232), generando un modelo de gestión de conocimiento para potenciar y mejorar la planificación de la unidad de análisis investigada. (ULEAM Extensión Chone) 
Uno de los resultados más importantes obtenidos en el tema que tratamos nos lo revela Naranjo López (2016) en su tesis doctoral donde hace una "Evaluación-acreditación de la educación superior en el Ecuador, metaevaluación y gestión académica de calidad" (Naranjo López, 2016) donde realiza un análisis de los conceptos y las posibilidades que brinda la gestión del conocimiento en la universidad. No obstante la percepción al tema, en nuestro país, que se considera más acertada a la correlación a nuestras variables se observa en los proyectos de Yachay, Ikiam, la UNAE y UNIARTES las cuales a decir del entonces ministro de educación superior es una "propuesta para la construcción de la sociedad del conocimiento y la creatividad" (Ramírez, 2013).

De manera general la UNESCO (2015) enfatiza en que América Latina y el Caribe presenta una "capacidad de adaptación muy limitada a los nuevos retos y desafíos que impone la globalización y la sociedad de la información en cuanto a la producción científica y la formación de capital humano" (UNESCO, 2015, p.139). La creación de nuevas empresas alejadas de la producción científica de las instituciones de educación superior se ha acrecentado en tanto las "nuevas" universidades o centro de educación superior surgen con apego a las labores docentes sin priorizar los posgrados y la investigación científica, menos un sistema de gestión de conocimiento que interrelacione y aporte valor (calidad) a los procesos propios de unos y otros.

\section{Conclusiones}

Como exigencia sine qua non para lograr el reto de generar una capacidad propia de producción científica y tecnológica además de mejorar la productividad científica básica y promover la investigación aplicada es crear un sistema gestión de conocimiento con cada una de las exigencias de la "sociedad del conocimiento". Los países en vías de desarrollo deben fomentar mediante estos sistemas una interrelación con instituciones de educación superior a nivel internacional sobre todo en materia de posgrados e investigación científica que nos permita contar con recursos humanos capaces de enfrentar los nuevos retos de la sociedad desde otra óptica.

La calidad en las instituciones de educación superior, será proporcional a la capacidad de respuesta de estas a los actuales y futuros retos y desafíos que le imponga la sociedad. Los sistemas de gestión de conocimiento coadyuva al acceso de la sociedad de la información, único camino para llevar al país a la sociedad del conocimiento y reducir las brechas de desigualdad además son indispensable para que estás instituciones mejoren su adaptabilidad al entorno y su capacidad de respuesta a los contextos imperantes.

La calidad de las actividades académicas es frágil debido a débiles sistemas de gestión del conocimiento y una baja inversión en ellos.

Pocas IES cuentan con documentos normativos y organizativos que sustente el trabajo documental, tampoco hay uniformidad ni estandarización en el tratamiento de la información lo que dificulta la gestión de conocimiento "especializados". Los procesos de integración de los conocimientos se deben realizar en el instante en que la información se introduce al sistema sin obviar el tan importante "valor añadido" de cada una de las demandas de los usuarios (Docentes, estudiantes o personal administrativo).

El desarrollo de las TIC's es un factor indispensable para la eliminación de la brechas de desigualdad en nuestras IES, el uso óptimo de estas tecnologías en el tratamiento de la información elimina diferencias e inequidades y aumenta la calidad de estas instituciones con independencia de la modalidad que ellas sigan. Está comprobada la incidencia directa de los sistemas de gestión de conocimientos en las economías de las universidades por lo que debe vérsele como una inversión y saber sacarle el máximo provecho, estos aportan valor y se convierten en una ventaja competitiva 
El modelo más utilizado, y el que proponemos para nuestras IES, es el de Ikujiro Nonaka y Hirotaka Takeuchi (como se citó en Huanca 2013), ya que promueve la creación del conocimiento desde el punto de vista epistemológico y ontológico y en el cual interactúan el conocimiento tácito y el explícito, nada más necesario para encontrar la calidad en la educación superior en nuestro país.

Es imprescindible tener en cuenta los sistemas de gestión de conocimiento para la creación de las "nuevas" universidades o centro de educación superior; debe ser tomado en cuenta a la par de las labores docentes, los posgrados y la investigación científica, estos son los encargados de las interrelaciones que aportan calidad a los procesos propios.

\section{Referencias Bibliográficas}

Álvarez Muñoz, P., \& Hernández Domínguez, C. (2016). Análisis longitudinal del Sistema Bibliotecario ecuatoriano. Revista Española de Documentación Científica.

Arch, X. (30 de diciembre de 2017). Innovating for Impact: The Next Evolution of Library Consortia. Collaborative Librarianship, 250-258.

Arraut, L. C., \& Gazabon, F. (2007). Modelo DECREXA para la Gestión del Conocimiento: Caso Concreto de Relación Universidad-Empresa en la Ciudad de Cartagena de Indias. Recuperado el 19 de Febrero de 2020, de OES Portal Educactivo de las Américas: https://recursos.portaleducoas.org/publicaciones/modelo-decrexa-para-la-gesti-n-delconocimiento-caso-concreto-de-relaci-n-universidad

CACES. (2 de Agosto de 2018). Ley Organiza de Educación Superior. Quito: LEXISFINDER.

CACES. (2020). Respuestas sobre el proceso de evaluación externa para los Institutos Superiores Técnicos y Tecnológicos en proceso de acreditación 2020. Quito: Consejo de Aseguramiento de la Calidad de la Educación Superior.

Consejo de Educación Superior. (2019). Reglamento de Régimen Académico. Quito: CES.

Correa Díaz, A. M., Arias Benjumea, M., \& Valencia Arias, A. (2019). La gestión del conocimiento: Una alternativa para la solución de problemas educacionales. Educare, 1-27.

Días, J. R. (2003). Modelo de Gestión del conocimiento aplicado a la universidad pública del Perú. Trabajo monográfico para optar por el titulo profesional de Licenciado. Lima, Perú: Universidad Nacional Mayor de San Marcos.

García, B. (30 de diciembre de 2016). Gestión del conocimiento desde el contexto universitario. Obtenido de Iberoamérica divulga: http://www.oei.es/historico/divulgacioncientifica/?Gestion-del-conocimiento-desde-elcontexto-universitario

Huanca, J. C. (21 de junio de 2013). Modelo de gestión de conocieminto para la gestión académica de las universidades públicas de Bolivia. Recuperado el 19 de Febrero de 2020, de Virtual educa. Colombia 2013: http://www.virtualeduca.info/fveduca/es/tematica-2013/91-modelosrec-tecnologicos-y-mecanismos-de-gestion/521-modelo-de-gestion-de-conocimiento-parala-gestion-academica-de-las-universidades-publicas-de-bolivia

Jama Zambrano, V. R. (2017). La Gestión del Conocimiento y su incidencia en la Planificación de la ULEAM. Extensión Chone. Revista Científica Dominio de las Ciencas, 232-249.

Jama Zambrano, V. R. (2019). Modelo de gestión del conocimiento para las instituciones de educación superior de la zona 4 del Ecuador. Recuperado el 18 de Junio de 2020, de Revista de Difusión cultural y científica de la Universidad La Salle en Bolivia: http://www.scielo.org.bo/scielo.php?script=sci_arttext\&pid=S2071081X2019000200008\&lng=pt\&tlng=es.

León Rodríguez, G. d. (2015). Gestión del conocimiento en la gerencia universitaria. Revista Científica Ecociencia, 15-29. 
Naranjo P, S., González H, D. L., \& Rodríguez M, J. (2016). El reto de la gestión del conocimiento en las instituciones de educación superior colombianas. Recuperado el 2020 de Junio de 11, de http://www.scielo.org.co/scielo.php?script=sci_arttext\&pid=S0123$48702016000200010 \& \operatorname{lng}=$ en\&tlng=es.

Naranjo López, G. (2016). Evaluación-acreditación de la educación superior en el Ecuador, metaevaluación y gestión académica de calidad. E-Prints Complutence. El repositorio de la producción académica en abierto de la UCM. Madrid, España: Universidad Complutense de Madrid.

Ramírez, R. (14 de agosto de 2013). Cuatro universidades son el pretexto simbólico para un pacto nacional para la construcción de la sociedad del conocimiento, creatividad y de la exelencia. Recuperado el 19 de Enero de 2020, de SENESCYT, Secretaría de Educación Superior, Ciencia, Tecnología e Innovación : https://www.educacionsuperior.gob.ec/la-era-delconocimiento-arranca-con-la-creacion-de-4-universidades-emblematicas/

RAUEC. (2016). Propuesta de indicadores para la evaluación institucional del Sistema de Educación Superior. Quito: CES.

UNESCO. (2009). Conferencia mundial sobre la educación superior - 2009: la nueva dinámica de la educación superior y la investigación para el cambio social y el desarrollo. Avaliação: Revista da Avaliação da Educação Superior (Campinas) (págs. 755-766). Paris: Sorocaba.

UNESCO. (2015). Situación Educativa de América Latina y el Caribe: Hacia la educación de calidad para todos al 2015. Santiago de Chile: Ediciones del Imbunche.

Urdaneta, A., Villasmil, M., Palacios Arrieta, A., \& Prieto Pulido, R. A. (2019). Gestión del conocimiento: Una concepción desde el enfoque sistémico. En Gerencia de las organizaciones. Un enfoque empresarial (págs. 81-114). Ediciones Universidad Simón Bolívar.

\section{Contribución del autor:}

\section{Autor}

Alexis Alfredo Tamayo Chacón

\section{Contribución}

Concepción y diseño, redacción del artículo y revisión del documento. 